\title{
MIX DEL PRODUCTO ÓPTIMO USANDO ALGORITMOS GENÉTICOS
}

\section{OF THE OPTIMUM PRODUCT USING GENETIC ALGORITHM}

\author{
Job Daniel Gamarra Moreno', Abraham Esteban Gamarra Moreno', Juan Gamarra Moreno
}

\section{RESUMEN}

Mix de producto óptimo significa determinar la cantidad de productos a producir para maximizar la ganancia. Para determinar el mix de producto óptimo de la Cooperativa Industrial Manufacturas del Centro de la ciudad de Huancayo, la empresa textil más importante de la región Andrés A. Cáceres, se ha construido el modelo híbrido que combina la simulación de eventos discretos y con los algoritmos genéticos. La simulación de eventos discretos se utiliza para inferir el costo unitario indirecto de cada producto debido al empleo de un sistema de costos basado en actividades. Para aplicar un sistema de costos basado en actividades se requiere información a posteriori, pero se puede conocerlo (aproximarlo) a priori aplicando la simulación de eventos discretos. Los algoritmos genéticos determinan el mix del producto óptimo que maximiza la utilidad. Estos algoritmos genéticos utilizan la codificación de valor para los cromosomas e incluyen técnicas para la solución de problemas con restricciones lineales. El mix de producto óptimo obtenido con el modelo disminuye las pérdidas con respecto al mix utilizado en el primer semestre, de aquellos productos cuyo costo unitario es superior a su precio, en un $43 \%$ e incrementan la utilidad en $123 \%$.

Palabras claves: Algoritmos genéticos, mix del producto, simulación de eventos discretos, costo basado en actividades.

\section{ABSTRACT}

Optimal product-mix means to determine the quantity of products to produce to maximize the gain. To determine the optimal product-mix of the Cooperativa Industrial Manufacturas del Centro from Huancayo city, the most important textile company in the region Andrés A. Cáceres, it has been built a hybrid model combining the discrete-event simulation and the genetic algorithms. The discrete-event simulation is used to infer the indirect unitary cost of each product due to the use of a system activity-based costing. To apply a system activity-based costing the posteriori information is required to, but one can know it (to approach it) a priori applying the discrete-event simulation. The genetic algorithms determine the optimal product-mix that maximizes the utility. These genetic algorithms use the code of value for the chromosomes and they include techniques for the solution of problems with lineal restrictions. The optimal product-mix obtained with the model decrease the losses of the product-mix used in the first semester, of those products whose unitary cost is greater than its price in $43 \%$ and they increase the utility in $123 \%$.

Keywords: Genetic algorithm, product-mix, discrete-event simulation, activity-based costing.

\section{INTRODUCCIÓN}

En los problemas de optimización (maximización o minimización), tratados ampliamente en la investigación de operaciones, ha surgido la posibilidad de utilizar los algoritmos genéticos no sólo en los problemas ya conocidos y satisfactoriamente solucionados, sino que además en problemas de mayor complejidad.

El modelo para determinar el mix de producto optimo (cantidad de productos a producir para maximizar la ganancia) de la Cooperativa Manufacturas del Centro, obtiene el mix de producto óptimo cuando el sistema de gestión de costos esta basado en actividades.

El modelo híbrido para determinar el mix del producto óptimo tiene como objetivo encontrar el mix de producto que maximice las utilidades, para lo cual usa la simulación de eventos discretos para determinar los costos indirectos y utiliza los algoritmos genéticos para obtener el mix del producto óptimo.

Tanto los algoritmos genéticos como la simulación de eventos discretos reducen la complejidad del problema y permiten su solución mediante un ordenador.

\section{MATERIAL Y MÉTODOS}

Según Santandreu (1), el costo basado en actividades (Activity Based Costing, ABC) es una herramienta estratégica y es considerada como herramienta financiera muy útil de la alta Gerencia. EI sistema de costo basado en las actividades tiene como principal función la de esta- blecer el grado de "Competitividad en el mercado". Asimismo, explica que este método analiza las actividades de los departamentos indirectos 0 soportes dentro de la organización para calcular el costo de productos terminados. Trata de solucionar de una manera bastante satisfactoria el problema de la asignación de los gastos indirectos de fabricación a los productos. Para determinar el costo de los productos o servicios utiliza el esquema de la figura 1.

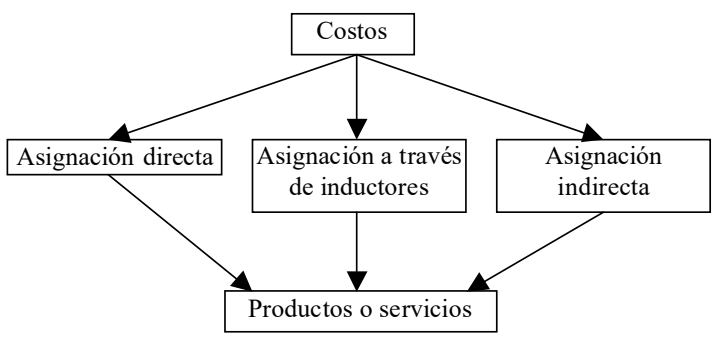

Figura 1.

\footnotetext{
${ }^{1}$ Facultad de Ingeniería de Sistemas, Universidad Nacional del Centro del Perú - Huancayo
} 
El modelo se ha dividido en dos módulos (figura 2): Definición de productos, actividades, inductores y restricciones; y el módulo para determinar el mix del producto.

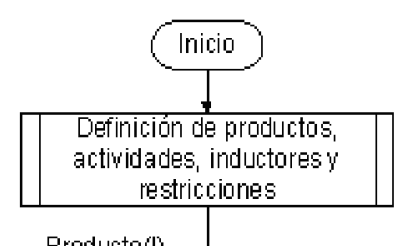

Volumen mi rimo de produc: iónd volumen lì ámime de produccióndh

Costo unitario objetiwoth

Costo unitario directoth Actividadidi

Costo tontal actividad(d) Inductoril

Consumo total inductor(d)

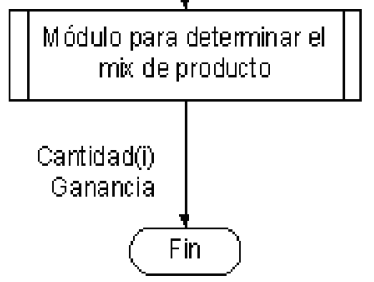

Figura 2. Diagrama de flujo del modelo. En el primer módulo se define los productos, las actividades que son necesarias para fabricarlos y los inductores con lo que se mide su consumo. Las actividades son las mismas que se utilizan en el sistema de costo basado en actividades. Es decir que cada actividad tiene su inductor de costo y su distribución estadística al que se ajusta el consumo de la actividad o su consumo fijo. Los productos se indican con el índice I y las actividades con el índice $J$, es así, que el Inductor $(\mathrm{I}, \mathrm{J})$ significa el inductor de la actividad $\mathrm{J}$ relacionada con el producto I. Por la característica del sistema de costo, cada actividad tiene asignado uno y sólo un inductor de costo.

Entre las restricciones están el costo objetivo de cada producto, el volumen máximo y mínimo de producción. El costo objetivo de cada producto es fijado de acuerdo con el precio de mercado y el margen de beneficio estimado. Si se trata de un nuevo producto que se desea lanzar, puede fijarse como precio en el mercado el mismo de la competencia. La idea clave del costo objetivo es la de estimar el precio que los consumidores estarían dispuestos a pagar por un cierto producto y deducir de ahí el beneficio.

El segundo módulo utiliza los algoritmos genéticos para determinar el mix de producto que genere la máxima ganancia. Para el algoritmo genético se necesario definir los siguientes parámetros: 3 probabilidades de cru- ce (3 operadores de cruce), probabilidades de mutación (3 operadores), la población y la generación cuando debe terminar las iteración. Es decir, para este caso la condición de terminación es cuando se alcanza la generación 1000, basado en el algoritmo genocop propuesto por Michalewicz (2).

\section{RESULTADOS}

Los datos se tomaron del estudio para la "Determinación de la Información técnica para la implementación de costos $A B C$ " para la Cooperativa Manufacturas del Centro del Perú, realizado por el Ing. R. Carhuamaca, el Año 2001 al 2002 (3) Estos datos tienen un horizonte temporal de un mes y por esa razón se ha trabajado con un mes de fabricación.

Los productos considerados tienen la presentación de la tabla 1 (sólo para tres productos)

Las actividades que incurren en costos indirectos se muestran en la tabla 2.

Los productos están relacionados con las actividades y estos a su vez con los inductores que son ajustados a una distribución estadística, en la tabla 3 se muestra los datos mencionados.

\begin{tabular}{|l|l|l|l|r|}
\hline $\begin{array}{l}\text { Denominación } \\
\text { producto }\end{array}$ & $\begin{array}{l}\text { Promedio } \\
\text { mensual del } \\
\text { costo unitario } \\
\text { directo (S/.) }\end{array}$ & $\begin{array}{l}\text { Promedio } \\
\text { mensual del } \\
\text { costo unitario } \\
\text { objetivo (S/.) }\end{array}$ & $\begin{array}{l}\text { Volumen máxi- } \\
\text { mo de produc- } \\
\text { ción mensual } \\
\text { (unidades) }\end{array}$ & $\begin{array}{l}\text { Volumen míni- } \\
\text { mo de produc- } \\
\text { ción mensual } \\
\text { (unidades) }\end{array}$ \\
\hline Paño g 600 & 10,00 & 32,00 & 8000 & 3000 \\
\hline Paño g 550 & 8,00 & 29,00 & 5500 & 2750 \\
\hline Paño g 400 & 6,00 & 28,00 & 10000 & 5000 \\
\hline
\end{tabular}

Tabla 1. Lista de productos

\begin{tabular}{|l|l|l|l|l|}
\hline $\begin{array}{l}\text { Denominación de } \\
\text { la actividad }\end{array}$ & $\begin{array}{l}\text { Descripción de } \\
\text { la actividad }\end{array}$ & $\begin{array}{l}\text { Promedio } \\
\text { mensual del } \\
\text { costo total } \\
\text { de la activi- } \\
\text { dad (S/.) }\end{array}$ & $\begin{array}{l}\text { Denomina- } \\
\text { ción del } \\
\text { inductor }\end{array}$ & $\begin{array}{l}\text { Descripción } \\
\text { inductor }\end{array}$ \\
\hline $\begin{array}{l}\text { Investigar el merca- } \\
\text { do }\end{array}$ & $\begin{array}{l}\text { Búsqueda de } \\
\text { nuevos nichos de } \\
\text { mercado }\end{array}$ & 23895,40 & $\begin{array}{l}\text { Número de } \\
\text { horas }\end{array}$ & $\begin{array}{l}\text { Mide la cantidad de } \\
\text { horas de investiga- } \\
\text { ción de mercado } \\
\text { usadas para un pro- } \\
\text { ducto }\end{array}$ \\
\hline $\begin{array}{l}\text { Enviar productos a } \\
\text { los clientes }\end{array}$ & $\begin{array}{l}\text { Entrega de pro- } \\
\text { ductos a los clien- } \\
\text { tes mayoristas }\end{array}$ & 100754,37 & $\begin{array}{l}\text { Número de } \\
\text { envíos }\end{array}$ & $\begin{array}{l}\text { Mide la cantidad de } \\
\text { envíos de un producto }\end{array}$ \\
\hline Promover ventas & $\begin{array}{l}\text { Visita a clientes } \\
\text { potenciales }\end{array}$ & 49784,64 & $\begin{array}{l}\text { Número de } \\
\text { horas }\end{array}$ & $\begin{array}{l}\text { Mide las horas usa- } \\
\text { das para visitar a un } \\
\text { cliente ofreciendo un } \\
\text { determinado producto }\end{array}$ \\
\hline Publicitar productos & $\begin{array}{l}\text { Contrata y diseña } \\
\text { las pautas publici- } \\
\text { tarias }\end{array}$ & 79692,34 & $\begin{array}{l}\text { Número de } \\
\text { avisos }\end{array}$ & $\begin{array}{l}\text { Mide la cantidad de } \\
\text { avisos publicitarios } \\
\text { para promocionar un } \\
\text { determinado producto }\end{array}$ \\
\hline $\begin{array}{l}\text { Procesar pedidos } \\
\text { de los clientes }\end{array}$ & $\begin{array}{l}\text { Atención de los } \\
\text { pedidos de los } \\
\text { clientes }\end{array}$ & 39815,41 & $\begin{array}{l}\text { Número de } \\
\text { pedidos aten- } \\
\text { didos }\end{array}$ & $\begin{array}{l}\text { Mide la cantidad de } \\
\text { pedidos que fueron } \\
\text { atendidos para un } \\
\text { producto }\end{array}$ \\
\hline
\end{tabular}

Tabla 2. Lista de actividades con sus respectivos inductores

\begin{tabular}{|c|c|c|c|c|}
\hline $\begin{array}{l}\text { Denominación } \\
\text { producto }\end{array}$ & Denominación actividad & \begin{tabular}{|l|} 
Distribución \\
estadística
\end{tabular} & $\begin{array}{l}\text { Parámetro } \\
1\end{array}$ & $\begin{array}{l}\text { Parámetro } \\
2\end{array}$ \\
\hline \multirow{5}{*}{ Paño g 600} & Investigar el mercado & Uniforme & $\begin{array}{r}0 \\
\end{array}$ & 2 \\
\hline & Enviar productos a los clientes & Normal & 30 & 3 \\
\hline & Promover ventas & Normal & 12 & 3 \\
\hline & Publicitar productos & Uniforme & 0 & 3 \\
\hline & Procesar pedidos de los clientes & Normal & 21 & 3 \\
\hline \multirow{5}{*}{ Paño g 550} & Investigar el mercado & Uniforme & 0 & 1 \\
\hline & Enviar productos a los clientes & Normal & 32 & 3 \\
\hline & Promover ventas & Normal & 5 & 1 \\
\hline & Publicitar productos & Uniforme & 0 & 1 \\
\hline & Procesar pedidos de los clientes & Normal & 9 & 3 \\
\hline \multirow{5}{*}{ Paño g 400} & Investigar el mercado & Normal & 5 & 1 \\
\hline & Enviar productos a los clientes & Normal & 58 & 4 \\
\hline & Promover ventas & Normal & 10 & 3 \\
\hline & Publicitar productos & Uniforme & 0 & 2 \\
\hline & Procesar pedidos de los clientes & Normal & 17 & 2 \\
\hline
\end{tabular}

Tabla 3. Relación productos, actividades e inductores. 
La columna parámetro 1 y parámetro 2 hace referencia a los parámetros necesarios de la distribución estadística, así, para la distribución normal son la media y la desviación estándar, pero para la distribución uniforme son los limites superior e inferior.

La materia prima principal para la fabricación de los productos es la lana que es obtenida como lana grasienta y luego de ser lavada pierde $40 \%$ de su peso, la producción máxima de lana lavada que se puede obtener es de $120000 \mathrm{~kg}$ por mes. Después de seleccionarla se obtienen los siguientes tipos de lana: A/B y C"/D. En la tabla 4 se consigna la producción mensual promedio de los tipos de lana.

\begin{tabular}{|l|r|}
\hline Tipo lana & $\begin{array}{l}\text { Producción mensual promedio } \\
(\mathbf{k g})\end{array}$ \\
\hline A/B & 72000 \\
\hline C"/D & 12000 \\
\hline
\end{tabular}

Tabla 4. Producción mensual promedio de tipo de Lana.

La tabla 5 muestra la cantidad de lana por producto que se utiliza.

\begin{tabular}{|l|c|r|}
\hline $\begin{array}{l}\text { Denomina- } \\
\text { ción producto }\end{array}$ & $\begin{array}{l}\text { Tipo } \\
\text { Lana }\end{array}$ & \multicolumn{2}{|l|}{$\begin{array}{l}\text { Cantidad usada } \\
\text { (en Kg.) }\end{array}$} \\
\hline Paño g 600 & C"/D & 1,30 \\
\hline Paño g 550 & A/B & 1,04 \\
\hline Paño g 400 & A/B & 0,78 \\
\hline
\end{tabular}

Tabla 5. Tipo de lana usada por producto.

Para determinar las restricciones se considerado solamente la materia prima como la lana porque su acopio es una tarea lenta y a veces los proveedores no cumplen con los requisitos de calidad exigidos, esto debido a que la producción de lana en la región es una actividad artesanal. El resto de materia prima usada se obtiene con relativa facilidad. En la tabla 6 se muestran las restricciones del modelo.

\begin{tabular}{|l|c|c|c|l|l|}
\hline $\begin{array}{l}\text { Tipo de } \\
\text { Lana }\end{array}$ & $\mathbf{x 1}$ & $\mathbf{x 2}$ & $\mathbf{x 3}$ & \multicolumn{2}{|c|}{} \\
\hline A/B & & 1,04 & 0,78 & $<=$ & 72000 \\
\hline C"/D & 1,30 & & & $<=$ & 12000 \\
\hline
\end{tabular}

Tabla 6. Restricciones del modelo.

Con el modelo del Mix del Producto Óptimo se ha determinado utilizando el programa diseñado para este fin. Los resultados obtenidos se muestran en la tabla 7 , el costo indirecto unitario se determino utilizando la simulación discreta y el Mix del producto óptimo mediante los algoritmos genéticos.

\begin{tabular}{|l|r|r|r|r|}
\hline $\begin{array}{l}\text { Denominación } \\
\text { producto }\end{array}$ & $\begin{array}{l}\text { Costo unitario } \\
\text { directo (SI.) }\end{array}$ & $\begin{array}{l}\text { Costo unitario } \\
\text { indirecto (SI.) }\end{array}$ & $\begin{array}{l}\text { Costo unita- } \\
\text { rio objetivo } \\
\text { (S/.) }\end{array}$ & $\begin{array}{l}\text { Mix a producir } \\
\text { (unidades) }\end{array}$ \\
\hline Pañog 600 & 10,00 & 22,77 & 32,00 & 4431 \\
\hline Paño g 550 & 8,00 & 11,08 & 29,00 & 5500 \\
\hline Paño g 400 & 6,00 & 10,93 & 28,00 & 10000 \\
\hline
\end{tabular}

Tabla 7. Resultados del modelo.

\section{DISCUSIÓN}

Es posible aplicar este modelo a otros sistemas de producción cambiando los datos ingresados en el módulo uno. La selección de las actividades y los inductores a considerar, en el modelo, es un proceso subjetivo, por lo que requiere de mayor análisis para obtener resultados confiables.

\section{CONCLUSIONES}

El modelo del Mix del producto óptimo es un modelo híbrido, que combina la: simulación discreta y los algoritmos genéticos eficientemente. Los resultados de este modelo dan resultados satisfactorios que permiten optimizar la ganancia.

\section{REFERENCIAS BIBLIOGRÁFICAS}

1 Santandreu E. y Santandreu P. Cálculo de costos con el método ABC. España: Ediciones gestión 2000 S.A.; 2000.

2 Michalewicz Z. Genetic Algorithms + data structurese = evolution programs. New York: Springer-Verlag; 1996.

3 Carhuamaca R. Estudio para la: Determinación de la Información técnica para la implementación de costos ABC. Huancayo: Cooperativa Manufacturas del Centro del Perú; 2002.

E-mail: jgamarra@uncp.edu.pe 